\title{
Los derechos de las mujeres migrantes y la garantía dentro del Estado ecuatoriano
}

\author{
Fecha de recepción: 2021-10-01 • Fecha de aceptación: 2021-11-16 • Fecha de publicación: 2021-12-01
}

Mario Danilo Granja Camacho

Universidad Estatal de Bolívar

mgranja@ueb.edu.ec

https://orcid.org/0000-0003-4590-1788

\section{Resumen}

Los derechos humanos son equidades universales inherentes al ser. La movilidad humana es una manifestación social reconocida por versados desde perspectivas que abarcan materias sociales y políticos. En este sentido, las mujeres migrantes tienen derecho a ser respetadas, garantizándoles la convivencia y erradicando el sexismo y medidas estigmatizadoras. El objetivo de esta investigación es percibir las vulnerabilidades y riesgos relevantes en las etapas del ciclo migratorio de las féminas, con el fin de identificar desviaciones en diferentes escenarios ecuatorianos. El enfoque metodológico es mixto, de tipo exploratoria e interpretativa. Se evaluaron datos estadísticos relacionados a la variable migración, en conjunto con el cumplimiento de garantía de las mujeres migrantes en Ecuador. Asimismo, se obtuvo información basada en testimonios de vida de mujeres involucradas en hechos de vulnerabilidad. Los resultados denotan violación en los beneficios migratorios, debilidad del estado de derecho de las migrantes y falta de integridad física por parte del Estado en algunos ámbitos migratorios. Las conclusiones señalan que es fundamental para el Estado ecuatoriano delinear acciones correctivas estipuladas en la Constitución, para restituir ciertos beneficios que fortalezcan ordenada y productivamente, los derechos relacionados con la estadía en el territorio ecuatoriano sobre las mujeres en condición de migrantes. 


\begin{abstract}
Human rights are universal equities inherent to being. Human mobility is a social manifestation recognized by experts from perspectives that encompass social and political matters. In this sense, migrant women have the right to be respected, guaranteeing their coexistence and eradicating sexism and stigmatizing measures. The objective of this research is to visualize the relevant vulnerabilities and risks in the stages of the migration cycle of women, in order to identify deviations in different Ecuadorian scenarios. The methodological approach is mixed, exploratory and interpretative. Statistical data related to the migration variable were evaluated, together with the fulfillment of migrant women's guarantees in Ecuador. Information was also obtained based on life testimonies of women involved in acts of vulnerability. The results show violations of migration benefits, weakness of the migrant women's rule of law and lack of physical integrity on the part of the State in some areas of migration. The conclusions indicate that it is fundamental for the Ecuadorian State to outline corrective actions stipulated in the Constitution, to restore certain benefits that strengthen in an orderly and productive manner, the rights related to the stay in Ecuadorian territory of women migrants.
\end{abstract}

Keywords: social welfare, human rights, migration, migrant women, vulnerability. 


\section{Introducción}

La movilidad humana es un fenómeno social reconocido y estudiado por expertos desde perspectivas que abarcan materias del ámbito social y político (Morel et al., 2018). Con el pasar del tiempo, mencionan diferentes autores, que la globalización ha generado una serie de característica direccionadas hacia el progreso e innovación; impulsada por hechos históricos positivos y adversos, que han permitido que los seres humanos busquen mejorar sus condiciones o bienestar, arraigados por factores que influyen directamente los niveles socioeconómicos, educativos y culturales; sobre todo en países suramericanos.

En América Latina, de acuerdo con Acuña-Alfaro \& Khoudour (2020), el Programa de las Naciones Unidas para el Desarrollo (PNUD); han venido reportando cifras durante los últimos 19 años (20102019), donde se observa un incremento en materia de emigraciones que oscila en un $26 \%$, en gran parte derivado por la situación que están viviendo los pobladores venezolanos, el cual según las tendencias no va desaparecer pronto. En este sentido, Fries (2019) argumenta que toda esta situación deriva de causas donde se destacan la falta de oportunidades para acceder a educación de calidad sin costo, la creciente demanda de ofertas laborales, y una situación de inestabilidad política; ya sea por conflictos internos en la organización del Estado, o por la presencia de grupos subordinados que infunden pánico e incertidumbre a nivel socioeconómico, civil y militar.

Esto conduce a afirmar la presencia de situaciones que de alguna manera motiva a los ciudadanos bajo las características descritas, a impulsarse y desligarse de todo lo que posee en su país de origen, originando la salida de todos o la mayoría de los integrantes del grupo familiar, con la intencionalidad de lograr cambiar la realidad en la que se encuentran. No se podría decir que, esta situación sobre la toma de decisión en buscar nuevas oportunidades en otras latitudes, está solamente ligado a los varones pues; las cifras emitidas por la Comisión Económica para América Latina y el Caribe (CEPAL) (citado en Fries, op. cit.) denotan que las mujeres se han encontrado de forma constante en los procesos migratorios, no obstante, en muchas ocasiones no fue tomado en cuenta las opiniones en debates y reflexiones sobre el tema. Adicionalmente, los abordajes epistémicos relacionadas a los problemas afines a la migración, han ocultado a las féminas como entes relevantes, debido a denuncias donde se determinan tratamientos inapropiados, solamente por el simple hecho de ser mujer o por discriminación de género.

No obstante, este enfoque ha cambiado debido al aumento notable de la presencia femenina en los movimientos migratorios, en los últimos 20 años. De hecho, en Latinoamérica, en los flujos migratorios la presencia de las mujeres en incremento es un rasgo característico, por ello, este hecho ha confirmado su incremento en las últimas décadas. Ahora, en tal sentido la feminización en el proceso de migración, no debe ser visto únicamente en cifras donde se evidencie incrementos de mujeres y su participación en situaciones migratorias, ya que, al mismo tiempo, al rol protagónico que se está volviendo más activo sobre los ámbitos económicos y sociales. Lo cual ha requerido superar contextos teóricos tradicionales sobre la actuación del hombre y la mujer; donde más bien se incorporen perspectivas basadas en el género, cuyo razonamiento lo que pretende es recalcar tanto experiencias como puntos diferentes que de forma común se enfrentan las féminas en los procesos de migración, considerando a los países de origen, y los de acogida. 
El cambio de paradigma, en este sentido ha sido tan notorio, en la actualidad las desempeñan algunas funciones como responsable principal, como de co-responsable de familias.

A pesar de esta costumbre arraigada en el pensamiento tradicional, es evidente, según Fajardo (2019), que la situación ha cambiado, ya que, en algunos casos, el papel del «jefe de hogar» ha sido reemplazado por las mujeres, quienes asumen responsabilidades diversas y desconocidas, para abrirse paso en los distintos grupos sociales y económicos. Esta situación conduce a que las mujeres se enfrenten a diversos escenarios, tanto en países desarrollados, como en vía de desarrollo; donde esta realidad es evidente cuando se ahonda en datos recopilados acerca del estilo y calidad de vida acorde con las políticas a las que cada lugar está regido.

Al respecto, Benavides \& Chávez (2014) mencionan que la cantidad de mujeres que optan por emigrar se encuentra en etapa de desarrollo y crecimiento, llegando a representar un $46 \%$, según los registros de entidades gubernamentales ubicadas en territorios limítrofes (fronteras), bien sea por vía terrestre o aéreo, en especial, en países que constan en la Comunidad Andina tales como: Bolivia, Colombia, Ecuador y Perú; dejando en evidencia que la falta de recursos económicos no es una limitante o impedimento para probar suerte en otro territorio, que le brinde nuevas oportunidades y mejoras para todo el grupo familiar y, de esta manera, obtener una calidad de vida que los dignifiquen como seres humanos.

Cada mujer residente en un lugar nuevo trae consigo la esencia de su país de origen, así como sus costumbres y tradiciones que la van a estar acompañando en todas las actividades que realice (modos de vidas). Investigaciones como las realizadas por Álvarez (2020) dejan entrever lo que ha sido el impacto a nivel psicológico de una persona, al empezar una vida diferente. El autor manifiesta que la soledad y la depresión son anomalías típicas durante una etapa de adaptación. Sin embargo, cuando las viajeras escogen su destino, internamente y de forma automática, se crea u origina una falta de conocimiento acerca de las leyes, reglamentos y estatutos que amparan a los individuos en esta condición. Esto significa, que no cuentan con un respaldo legal que les permita exigir sus derechos, así como cumplir sus obligaciones, convirtiéndose en víctimas relacionadas sobre todo a nivel laboral (se aprovechan los patrones de su condición de necesidad), sexual y discriminación racial, o lo también conocido como xenofobia.

A todas estas, el Estado ecuatoriano es una de las Repúblicas en las que constitucionalmente muestra una figura donde las mujeres son reconocidas como sujetos de derecho, es más, cuenta con apartados en los que se reflejan y detallan claramente las cláusulas y definiciones en las cuales destacan que una persona puede llegar al país en calidad de refugiada o emigrante, y puede optar por tener los mismos derechos y beneficios de los habitantes ecuatorianos, siempre y cuando se encuentra en calidad de regular a nivel de procesos migratorios, dicho de otra forma, que cumpla con la documentación (visa) apropiada. En este punto, Flores (2020) destaca que el asesoramiento en torno a los protocolos a seguir para legalizar la estadía de mujeres extranjeras y sus familias, es una problemática que se ha venido agudizando durante los últimos cinco años, sobre todo producto de la crisis socioeconómica y política que están viviendo miles de personas en Venezuela, que ha generado expansión migratoria, sobre todo en el Ecuador. 
Las condiciones socioeconómicas y de salud con las que llegan las mujeres migrantes al territorio ecuatoriano resultan un factor condicionante para emprender un camino diferente; es decir, esto produce un cambio donde se puedan abrir nuevas oportunidades para la población, debido al grado de preparación y conocimiento que pueda tener una mujer donde se pueden diversificar ciertos rubros en el plano comercial, educativo y tecnológico. Lo resaltante en este punto lo destacan Salazar et al. (2019), quienes alegan que se abre un abanico de posibilidades de empleabilidad, siempre y cuando se hayan homologado los títulos, en este caso, frente a la Secretaría de Educación Superior, Ciencia, Tecnología e Innovación (SENESCYT) quienes representan el organismo autorizado por el Estado, para avalar el grado de preparación. Por el contrario, hay que destacar la presencia incesante de casos xenofóbicos debidamente reportados por las mujeres migrantes, donde se evidencian ciertas trabas y rechazos durante el desarrollo de estas actividades protocolares.

Aunque el tema de los abusos generados por una condición de vulnerabilidad por ser mujer migrante, ha ocasionados problemas que trastocan casos de violencia psicológica o sexual, el Estado ecuatoriano, a través de sus diferentes poderes, trabaja por mantener la paz y garantizar una vida digna a todas las personas que se asientan en el país. Una revisión del marco jurídico vigente en Ecuador emprendidas por Salazar et al. (2019) (op. cit.) permite conocer cuáles son las políticas públicas de gran relevancia, al ser un país de acogida a personas con un interés, en que se les brinde protección internacional por las condiciones de inseguridad o conflicto vividas en sus países natales, como es el caso de mujeres colombianas o peruanas.

Partiendo del anterior antecedente, al momento de garantizar el ejercicio de derecho de las mujeres migrantes en el Ecuador, es preciso mencionar la importancia emanada del Art. 14 de la Declaración Universal de Derechos Humanos, indicando en caso de persecución, que toda persona tiene derecho a buscar asilo. En 1951, la Convención de la Organización de Naciones Unidas (ONU) sobre los refugiados, así como también su protocolo redactado en 1967, tienen como objeto proteger a las personas refugiadas en una situación de expatriación, siempre y cuando se encuentren en riesgo. Asimismo, y de forma más amplia e integral, en 1990 se reunieron la convención sobre derechos de los trabadores migrantes, apalancándola como una herramienta amplia para toda persona refugiada y su familia (Benavides \& Chávez, 2014).

La generación de un clima de intolerancia hacia mujeres migrantes afecta negativamente y vulnera derechos inherentes que cada una posee de manera universal. El género femenino, sigue siendo uno de los factores determinantes para desencadenar una serie de eventos, en los cuales la falta de aplicación de los derechos es reconocida. Cabe destacar, que en función de la jerarquía del poder legislativo, se encuentra la Constitución de la República del Ecuador (Asamblea Nacional del Ecuador, 2008) vigente; el cual, por ejemplo, al ser comparada con la Constitución de La República Bolivariana de Venezuela; se denotan semejanzas en aspectos basados en precautelar los principios de las relaciones internacionales declaradas en el artículo 416 de la del Ecuador, el cual señala que "Las relaciones del Ecuador con la comunidad internacional responderán a los intereses del pueblo ecuatoriano, al que le rendirán cuenta sus responsables y ejecutores, y, en consecuencia: exige el respeto de los derechos humanos, en particular de los derechos de las personas migrantes, y propicia su pleno ejercicio mediante el cumplimiento de las obligaciones asumidas con la suscripción de instrumentos internacionales de derechos humanos". 
En este sentido, este estudio analiza las deficiencias en la legislación ecuatoriana en torno a la realidad que viven las mujeres migrantes en condición de vulnerabilidad.

\section{Metodología}

El enfoque de esta investigación es mixto, realizando un alcance que combina dos tipos de investigación jurídica, el primero es de tipo exploratoria, debido a que los datos estadísticos relacionados a cifras bajo la variable migración, en conjunto con el cumplimiento de la garantía de las mujeres migrantes en nuestro territorio, no cuenta con información actualizada (Tantaleán, 2015). El material complementario del cual se pudo recabar información de primera mano, proviene de una muestra pequeña de la población, basado en la hermenéutica de trabajos recopilados de bibliotecas virtuales de alto impacto en la rama de Derecho; es decir, son testimonios de vida sobresalientes de mujeres que se vieron involucradas en hechos en los cuales sus derechos integrales, fueron vulnerados por parte de sus semejantes.

La otra arista del estudio se ampara en la descripción de la normativa vigente y sus modificaciones por parte de las autoridades competentes en materia de derecho internacional, como en el manuscrito de Luque et al. (2019), en el mismo que la recopilación de información ha sido comparada entre países que mantienen relaciones hermanas con reseña histórica, como son los miembros de la Comunidad Andina, al integrar de manera paulatina a la mujer en apartados de la Carta Magna de cada uno.

Con base a ciertos datos obtenidos de estudios preliminares de la Comisión Económica para América Latina y el Caribe (CEPAL) (citado en Fries, 2019), se notó el incremento de una feminización migratoria, así como la revelación de problemas biológicos, políticos y sociales, que son el resultado de la movilidad humana constante, sin tomar en cuenta medidas necesarias para su prevención.

\section{Resultados}

Al momento de analizar los testimonios, del cual se tomó en consideración para delinear las deficiencias en la legislación ecuatoriana en torno a la realidad que viven las mujeres migrantes en condición de vulnerabilidad, se presentaron y crearon algunas categorías que ayudaron a ir consolidando esas inconsistencias que presenta el proceso sobre la migración y trato en las mujeres. Los movimientos migratorios originados en Ecuador obedecen a una multiplicidad de causas socioeconómicas, políticas, educativas, de sustentabilidad, de conflictos personales, curiosidad, sentido de supervivencia, buscando un mejor nivel de vida, entre otros; en gran parte de los casos, la población que ha optado por la migración en su país de origen, ha estado expuestas a riesgos que han generado inseguridad, bienestar y sus medios de sustento, lo que irremediablemente acota sus alternativas de vida.

Así, se determinó que la desigualdad socioeconómica configura como elemento principal en la toma de decisiones para migrar, optando por buscar condiciones mejores de vida incluyendo una mejora laboral. Migrar, visto desde una perspectiva de la mujer, por lo general se encuentra 
vinculada a salidas de problemas personal, incluyendo aspectos de pareja y familia, además de violencia o incomodad con la comunidad o región en donde residen los migrantes. Un dato que a veces se olvida y tiene mucha relevancia en los procesos migratorios es las ocurrencias o desastres naturales, estas situaciones puede ocasionar pérdidas tierras, viviendas, entornos de trabajo, entre otros aspectos, del mismo modo, puede desestabilizar la situación económica, limitando incluso el acceso a servicios básicos, por ello, todas estas situaciones pueden ocasionar que se presente una migración internacional o interna como una nueva alternativa de vida.

Estas diversas situaciones mencionadas evidencian la relevancia de una perspectiva multicausal y amplia sobre el tema migratorio. De hecho, cuando las personas se refieren sobre la decisión tomada de emigrar, en primera instancia confirman lo difícil que ha representado haber dejado toda su vida atrás (material y humana) e insertarla en una maleta donde apenas pueden traer solo lo estrictamente necesario. Comenzar de cero siempre ha sido considerada un reto interesante, pero desde una postura migratoria es bastante diferente, ya que, en el caso de las mujeres, tienen que sortear situaciones adicionales (discriminación, abusos verbales y sexuales, exclusión social), que realmente le agregan un plus adicional que hace más cuesta arriba la posibilidad de estabilizarse desde lo económico, social y educativo. Considerando la situación, es necesario tomar en cuenta a la migración no solo por cuestiones individuales, es necesario contener las situaciones en la sociedad globalizada lo cual va de mano con las motivaciones, esto genera lo que se conoce como la diversidad migratoria.

Tomando en consideración la vida de las mismas mujeres migrantes y las políticas públicas, en este caso ecuatoriana, según las realidades que viven las féminas existen contradicciones e inconsistencia en lo expresado en las leyes, como por ejemplo, de movilidad humana, donde se busca estabilizar situaciones de regularidad que tienen que ver con asilos, refugios, otorgamiento de visas temporales y permanentes; pero el mismo sistema, o en su defecto las personas encargadas de realizar estos trámites, se convierten en obstáculos para las mujeres migrante que no logran obtener su regularidad migratoria. En este sentido, es bueno destacar que las situaciones son diferentes cuando una persona migra con intenciones de estabilizarse en un nuevo lugar, que la persona que lo realiza de manera temporal, pero de forma continua como es el caso de trabajadores del campo, los cuales viajan por fronteras en temporada de cosecha, de forma similar, las personas que residen en las fronteras quienes tienen que cruzar de forma diaria para trabajar o estudiar y de la misma forma retornar al lugar de origen.

Así como también, no lo es quien migra con tiempo delimitado, como por ejemplo las personas que viajan por fines educativos. De forma similar, es diferente la situación de las personas que migraron por un periodo de tiempo prolongado, cada situación suscitada genera retos como inclusión y protección en el ámbito laboral y social. Adicional a lo expuesto hasta este punto, se puede identificar diferentes retos, situaciones y riesgo que atraviesan las féminas migrantes, sin embargo, se debe diferenciar la condición del país de origen, proceso transitorio y el lugar de destino, para organizar analíticamente se puede hacer uso del ciclo de migración, así se puede diferenciar las fases y la vulnerabilidad de las mujeres en la situación migratoria, por ello, los requerimientos de políticas públicas de protección social están enmarcados en la siguiente Figura 1. 
Figura 1.

Ciclo de la migración

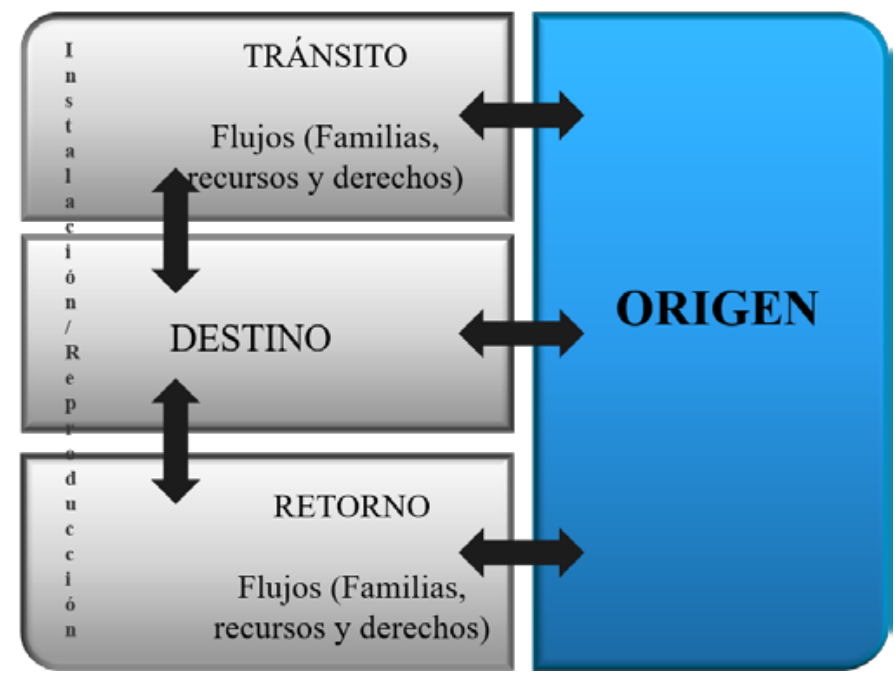

Según la información recolectada, el proceso de migración, es comprendido como sistema para la sociedad, el cual inicia lugar de origen, con las motivaciones e intereses individuales, en este caso a las mujeres a migrar. Seguidamente se fija o selecciona el lugar de destino a alcanzar, tomando en consideración el análisis de alternativas de financiamiento y acomodos familiares para su concreción, incluyendo la búsqueda de mayor protección social por vías formales e informales. Esta parte inicial, pertenece a un posible plan pensado, cuyo basamento se centra en el planteamiento de una estrategia de mediano y largo plazo; con la voluntad de concretar una movilización de forma permanente y con diversos recursos individuales, como consecuencia, puede ser resultado de una decisión considerada como forzada, frente a situaciones adversas o excepcionales del lugar de origen.

Comenzado el proceso, existe una fase intermedia de tránsito que, dependiendo del lugar de destino, se traduce y convierte en una panacea de riesgos y vulnerabilidades. Pueden existir variaciones de la duración de esta fase, sobre todo frente a las complicaciones legales y geográficas, así como también, la presencia de ambientes hostiles para llegar al destino final. Lo mencionado, abarca posibilidades de que sea permanente traduciendo a este punto las necesidades de inserción tanto social como económica en un lugar distinto al original, o a su vez, un retorno de forma eventual al lugar de origen frente a un posible fracaso en la meta planteada. En cualquier situación, en condición de vulnerabilidad potencialmente considerada, se plantea a la supervivencia y a la seguridad física como desafíos inmediatos, sobre todo para las mujeres.

Ahora bien, el tema de la vulnerabilidad migratoria de las mujeres en el territorio ecuatoriano, en cierta forma concuerda con lo establecido por la CEPAL (2016), en este punto se encuentran factores sociodemográficos, los cuales son aspectos potenciales para generar fuentes de vulnerabilidad durante el proceso migratorio. Además, son considerados como predictores de necesidades para el sistema de protección social, punto que es relacionado con aspectos de 
desigualdad social en la región como son la condición socioeconómica, el género, el ciclo de vida, la raza y la etnia y el territorio, pero también, son consideradas la estructura familiar de migrantes.

De forma similar, factores como el índole político-institucional y de mercado de trabajo involucran a la vulnerabilidad sobre el bienestar de las mujeres migrantes, a lo cual incide en la condición migratoria de la persona y la inserción en el mercado de trabajo. Respecto a la condición migratoria, existen dos vías posibles, la primera es la condición regular, aquí se encuentran los migrantes en calidad de refugio, cuando se habla de una condición regular, involucra disponer de un estatus en el lugar de llegada para optar a una residencia, involucrando así la libre circulación y el ejercicio de actividades y el uso de derechos. Por otro lado, la segunda vía es la condición irregular, lo cual involucra a personas indocumentadas, en este aspecto existe una vulnerabilidad estructural adicional y de índole netamente legal.

En referencia, a la inclusión de mujeres migrantes en el campo laboral, involucra el acceso a mecanismos de inclusión y derechos, sobre todo cuando la actividad laboral es formal. A partir de estos razonamientos epistémicos y empírico, se formularon una serie de categorías intervinientes, que se originaron tomando en consideración la participación de las mujeres que han vivido de cerca las diferentes vulneraciones que se producen, bien sea al momento del tránsito, como una vez llegado a su lugar escogido como nuevo hogar, en búsqueda de esa estabilidad socioeconómica, emocional, psicológica y educativa; que no pueden obtener en su país de nacimiento.

Las mujeres migrantes, al trasladarse de forma individual o con compañía, van acompañadas de motivaciones e involucran aspectos sociodemográficas; que llevo a plantear los siguientes factores de vulnerabilidad y desigualdad para las mujeres migrantes: partiendo desde la identificación de una dimensión territorial, se muestran factores sociodemográficos, tales como género femenino, ciclo de vida, raza (afrodescendientes), etnia (indígenas), estructura familiar, hábitos unipersonales y la condición socioeconómica (medido en pobreza). Ahora, desde la identificación de factores político-institucional y de mercado, se detallan el trabajo informal y la condición migratorio (irregularidad).

Estos factores que influyen en la vulnerabilidad de los derechos de las mujeres migrantes, se reflejan en el sistema de protección social que principalmente se genera por el ámbito laboral, donde la inclusión al campo de trabajo es relevante. Aquí el Estado tiene la función de ofrecer apoyo para iniciar la vida laboral, regulando las relaciones laborales e incluso diseñando mecanismos no contributivos, con el fin de garantizar derechos a migrantes que no cuenten con un mercado laboral formal. Desde otra perspectiva, la realidad es contraria, por lo que se hace necesario emplear medidas de auditoría donde el objetivo central sería respetar los derechos de las mujeres migrantes en este sentido. Esta situación se ubicó dentro de la etapa del ciclo de vida aun cuando no todas las decisiones migratorias se encuentran enlazadas a la búsqueda de mejores oportunidades de empleo.

Como se puede observar, existen rasgos distinguibles, que afectan el estatus o vulneran los derechos de las mujeres migrantes, donde sobresalen la disminución y eventual abandono sobre el derecho al trabajo, que solo conduce a elevar la precariedad y el deterioro, en este caso, de 
las mujeres que buscan establecerse en un país distinto. Consecutivamente, la calidad de vida y el bienestar es el resultado de la fusión de la trayectoria laboral anterior, los activos físicos y monetarios acumulados, los beneficios proporcionados por el Estado en las etapas anteriores y actuales, así como también, la calidad del sistema de protección del lugar de residencia de las personas.

Este enfoque se relaciona directamente con el ciclo de vida, lo cual es direccionado a las necesidades y niveles de riesgo que se puede diferenciar en las distintas etapas del ciclo por parte de Estado ecuatoriano. En ciertas situaciones, la migración involucra riesgos a la vida de los migrantes, en Ecuador, un problema estructural es la pobreza varios sectores. La pobreza evidenciada en la población rural fue el principal detonante de los flujos migratorios hacia zonas urbanas. Los comentarios emitidos por las mujeres han demostrado que las poblaciones en extrema pobreza no optarían por migrar a otro país, debido al costo elevado que involucra la migración, entendiendo así, que las personas que optan por migran invierten una cantidad fuerte de dinero.

En este sentido, el sexo femenino es vulnerable, supone tener ingresos insuficientes e inestables, siendo contradictorio a lo establecido en la Constitución de la República del Ecuador, respecto a la igualdad y no discriminación de ningún tipo. El Estado debe comprometerse y tomar decisiones respecto a este punto migratorio, en búsqueda de que se haga cumplir lo estipulado en la Carta Magna y poder dignificar este tan maltratado sector poblacional. Es de destacar, que uno de los datos recolectados e interpretados en este tema migratorio de la mujer, es que la mujer es cabeza de hogar lo cual ha incrementado de forma considerable. Por ello, los cambios se están en gran parte direccionado a la consolidación de las mujeres en el ambiente laboral.

Por lo establecido, se puede considerar la predominancia de la migración de mujeres y que el Estado ecuatoriano debe aprovechar, para afianzar esta coyuntura, que en muchas ocasiones tales derechos o vulnerabilidades, las cometen personas que por alguna razón tienen o ha tenido problemas desagradables con un extranjero. Por ello, la migración no implica la desintegración del vínculo familiar, pero genera modificación en la estructura de residencia de la familia, así como desafíos para el cuidado de los miembros dependientes. Se puede destacar, la diversificación del modelo tradicional familiar, fundamentándose en investigaciones de migración en Ecuador, se puede reconocer características centrales del modelo tradicional, direccionándose a la división del rol de género y la estructura fundada en los hijos durante su fase dependiente.

En función a lo descrito en el epígrafe anterior, un eje estructurante de la desigualdad social es el género en Ecuador en relación a la situación de migración. El incremento de la feminización es una tendencia común en el proceso migratorio, de modo que, en el caso de las mujeres migrantes, se requiere tomar en consideración a las vulnerabilidades basadas en el género, puntos que van en incremento durante la migración. Al respecto y, aprovechando los factores que se crean alrededor de los riesgos y vulneraciones de la mujer migratoria, cabe mencionar las siguientes categorizaciones que complementan todo lo hasta ahora expresado.

La desinformación, el cual tiene relación con situaciones legales transitorias que tienen que ver con la condición irregular en el país, que al mismo tiempo pudiera ser considerada como exclusión 
social. También se destaca las dificultades para obtener permisos oficiales y a nivel familiar, cuyo objeto transitorio se centra en tener acceso o no a la protección social, que debe ser de tipo gratuito o que el Estado ecuatoriano proporcione a todo aquel que se encuentre en el territorio nacional. Esta categoría produce una estigmatización y genera persistencia de riesgos y carencias en el lugar de origen. Asimismo, se destaca la limitación en cuanto al acceso a documentos de viajes, que resulta o se condiciona con la categorización anterior, sobre todo cuando se toca el tema de la salud. Se convierte en un obstáculo vulnerable que inclusive no permite la regularización y permanencia legal en el país.

Luego de todo esto se mencionan categorías que tienen relación directa con la violencia (doméstica, social y sexual) y aspectos sociales que realmente lo que producen es acrecentar los problemas migratorios de las mujeres. En este caso, el Estado ecuatoriano debe tener la capacidad para reinsertar a las féminas en los diferentes campos, donde estas se encuentren capacitadas, sin distinción de situación migratoria. Es contribuir de alguna manera a hechos de dignidad de los seres humanos, y más a las mujeres, quienes representan la vida, el amor y el compañerismo.

Una categoría adicional asociado a las mujeres migrantes, es la discriminación en el mercado laboral. Entre las manifestaciones rescatadas en la investigación, se encontraron marcadas diferencias salariales con las mujeres y la segregación profesional en actividades precarias y sin un buen reconocimiento salarial como es el caso del trabajo doméstico. Por resultado, la protección social forma parte para del proceso para obtener una igualdad de género y de forma general.

Como se observa, la vulnerabilidad radica principalmente hacia la integridad física, la violación de los derechos humanos y laborales fundamentales durante el trayecto hacia el país de destino y al atravesar las fronteras, en especial cuando el traslado es irregular. Se destaca la extorsión o secuestro, riesgos de exceso psicológico, maltrato físico, abuso sexual, particularmente en el caso de mujeres. Este se potencia muchas veces con la inexistencia más o menos generalizada de políticas de acogida y atención a población femenino migrante en tránsito y la localizada.

\section{Conclusiones}

Toda política pública debe enmarcarse sin perder de vista la institucionalidad, cuyas características se enfilan en mantener un rol central sobre su eficacia, eficiencia, capacidad de inclusión y la garantía de los derechos ciudadanos de cualquier tipo. Una forma de lograr lo anterior deviene de la identificación de sectores específicos relacionados con la política pública y social, que engloba el análisis situacional bajo cuatro dimensiones: la jurídico-normativa, organizacional (pluricultural y plurinacional), técnico-operativa y de financiamiento.

Estas dimensiones, en el caso de esta investigación, se centra en normativas nacionales dirigidas hacia la población migrante de mujeres, con el fin de demostrar ciertas vulneraciones hacia ese género que contrasta notoriamente con las estipulaciones observadas a nivel constitucional. Es necesario que el Estado tome en consideración la importancia de tener una legislación adecuada para responder a las necesidades, aspiraciones y las demandas de las mujeres migrantes y, que, a 
su vez, se vuelva un reflejo hacia el respeto de los derechos humanos, producido en gran parte por personas que no representan esos aires que el país desea para esa población femenina migrante. Este trabajo constituye un instrumento donde se plasman situaciones que deben ser revisadas y verificadas a nivel nacional por parte del Estado, con miras a favorecer la estabilidad, definición de criterios y procesos.

No obstante, todos estos vínculos en materia migratoria hacia las mujeres, deben explorarse constantemente, sin descuidar ningún espacio, por muy elemental que sea, en especial las normativas laborales, educación, vivienda, regularización legal migratorio y salud; debido a su aporte en la protección y reinserción social de las féminas. Mediante esta investigación se analizaron y visibilizaron las vulnerabilidades y riesgos más importantes en las distintas etapas del ciclo migratorio, con el fin de identificar medidas y estrategias relevantes en los diferentes y muy diversos escenarios migratorios ecuatorianos. Lo importante es que, las personas migrantes del sexo femenino se conviertan en un segmento poblacional sistemático, considerado por los sistemas de protección social en el país. No se trata de que las mujeres migrantes compitan con las ecuatorianas, sino que todas se vean beneficiadas en función de la titularidad de los derechos intrínsecos existentes.

La migración no debe ser sinónimo de sufrimiento y castigo para las mujeres, por el contrario, debe ser representativo de nuevas oportunidades en el bienestar personal y familiar. Muchas de estas féminas pueden ser consideradas como innovadoras y emprendedoras, ya que buscan una calidad de vida que, por razones especificadas a lo largo de este texto, no pudieron conseguir en sus países de origen. El desafío se debe centrar en la forma como el Estado puede aprovechar al máximo esta situación, creando nuevos conceptos de inserción social, pero al mismo tiempo, estableciendo normas serias para lograr organizar y ordenar este sector femenino en materia de migración de manera productiva, para así, poder disminuir o erradicar con las vulneraciones y prejuicios y resarcir los derechos humanos de estas guerreras migrantes. 


\section{Referencias}

Acuña-Alfaro, J., \& Khoudour, D. (31 de enero de 2020). El potencial de la migración en América Latina y el Caribe. Programa de las Naciones Unidas para el Desarrollo. PNUD. https://www1.undp.org/content/undp/ es/home/blog/2020/harnessing-the-potential-of-migration-in-latin-america-and-the-c.html

Álvarez, S. (2020). Ilegalizados en Ecuador, el país de la "ciudadanía universal" Sociologias, Porto Alegre, 22(54), 138-170. https://doi.org/10.1590/151745 22-101815

Asamblea Nacional del Ecuador. (2008). Constitución de la República del Ecuador. Decreto Legislativo 0. Registro Oficial 449 de 20-oct-2008. Estado: Vigente. https://www.oas.org/juridico/pdfs/mesicic4 ecu const.pdf

Benavides, G., \& Chávez, G. (2014). Migraciones y derechos humanos. El caso de la Comunidad Andina (CAN). Revista Científica General José María Córdova, 12(14), 75-93. https://doi.org/10.21830/19006586.57

Comisión Económica para América Latina y el Caribe. (2016). La matriz de la desigualdad social en América Latina. CEPAL.

Fajardo, T. (2019). El pacto mundial por una migración segura, ordenada y regular: un instrumento de soft law para una gestión de la migración que respete los derechos humanos. Revista Electrónica de Estudios Internacionales, (38). https://doi.org/10.17103/reei.38.02

Flores, M. (2020). Mujeres migrantes venezolanas: Entre políticas vetustas y cadenas de cuidados. Encuentros: Revista de Ciencias Humanas, Teoría Social y Pensamiento Crítico, (12), 75-90. http://doi.org/10.5281/ zenodo.3951224

Fries, L. (2019). Las mujeres migrantes en las legislaciones de América Latina. Análisis del repositorio de normativas sobre migración internacional del Observatorio de Igualdad de Género de América Latina y el Caribe. Santiago: Naciones Unidas (CEPAL). https://repositorio.cepal.org/bitstream/handle/11362/44655/1/ S1900271_es.pdf

Luque, A., Carretero, P., \& Morales, P. (2019). El desplazamiento humanitario en Ecuador y los procesos migratorios en su zona fronteriza: Vulneración o derecho. Espacios, 40(16), 1-13.

Morel A., Nuñez, D., \& Ramires, A. (2018). Migración y violencia contra la mujer migrante y refugiada. Novos Estudos Jurídicos, 23(1), 303-328. https://doi.org/10.14210/nej.v23n1.p303-328

Salazar, D., Cobo, A., Cruz, C., Guevara, M., \& Mesías, M. (2019). La fuerza vinculante de las Opiniones Consultivas de la Corte Interamericana de Derechos Humanos a la luz del derecho y la justicia constitucional en Ecuador. Foro, Revista De Derecho, (32), 123-143. https://doi.org/10.32719/26312484.2019.32.7 
Tantaleán, R. (2015). El alcance de las investigaciones jurídicas. Derecho y Cambio Social, 12(41), 1-22. https:// dialnet.unirioja.es/servlet/articulo?codigo $=5456857$ 


\section{Copyright (c) 2021 Mario Danilo Granja Camacho}

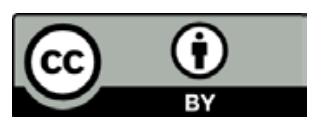

Este texto está protegido bajo una licencia internacional Creative Commons 4.0.

Usted es libre para Compartir-copiar y redistribuir el material en cualquier medio o formato

- y Adaptar el documento - remezclar, transformar y crear a partir del material-para cualquier propósito, incluso para fines comerciales, siempre que cumpla las condiciones de Atribución. Usted debe dar crédito a la obra original de manera adecuada, proporcionar un enlace a la licencia, e indicar si se han realizado cambios. Puede hacerlo en cualquier forma razonable, pero no de forma tal que sugiera que tiene el apoyo del licenciante o lo recibe por el uso que hace de la obra.

Resumen de licencia - Texto completo de la licencia 